\title{
Research on the Rough Extension of Ontology Description Language of SWRL
}

\author{
Xian-kun Zhang, Xin-ya Gao, Qian Zhang, and Jia Jia \\ College of Computer Science and Information Engineering, Tianjin University of Science and Technology, Tianjin 300222, China \\ Correspondence should be addressed to Xin-ya Gao; 43415838@qq.com
}

Received 18 November 2015; Accepted 27 January 2016

Academic Editor: Hui Cheng

Copyright ( 2016 Xian-kun Zhang et al. This is an open access article distributed under the Creative Commons Attribution License, which permits unrestricted use, distribution, and reproduction in any medium, provided the original work is properly cited.

Although ontology has strong ability to express knowledge, it is difficult to express uncertain or imprecise information using the language of ontology. In order to improve the ability to express uncertain information, this paper extends the Semantic Web Rule Language (SWRL) and gives the extension of reasoning rules. According to the rough ontology and the rules of SWRL, it updates the knowledge base. Firstly, the concept of rough ontology and the extension of rough relationship of ontology are put forward; secondly, it gives the extension method for concepts, relationships, axioms, examples, and rules of SWRL. Finally, a psychological counseling case shows that the method can well express the uncertainty of knowledge, and it is able to well express the reasoning rules.

\section{Introduction}

Ontology is "a formal specification of a shared conceptualization" [1]. It is an important means to describe the semantic knowledge model. Because imprecise knowledge is universal in real life, it is always the hot issue that the extension of ontology description language can express uncertain or imprecise knowledge in the academic circles. In order to solve the representation of imprecise or uncertain knowledge, the common method of the extension for imprecise ontology is to introduce the theory of fuzzy set and rough set for fuzzy ontology [2], rough ontology [3], or fuzzy rough ontology [4-6].

According to these three different imprecise ontology models, many scholars have put forward different methods for the extension of ontology description language. Hailong et al. [7] extend the language of OWL (Web Ontology Language) based on the fuzzy description logic F-ALC(G). Hongyun [8] analyzes classes, properties, examples, and axioms for rough ontology of the semantic Web through the definition of relevant rules. Xijin [9] introduces upper and lower approximation sets for rough set and puts forward the method to express rough fuzzy ontology of the semantic Web in OWL. These studies expand the ability to express imprecise knowledge in OWL.
OWL is on the basis of descriptive logic. At present, it is widely used by people. It makes OWL ensure the expressive ability of semantics and guarantees the decidability of reasoning at the same time. In recent years, a lot of research work has made it available in OWL ontology with different types of linguistic rules. To provide more powerful knowledge representation and reasoning ability, the work has provided some extensions of OWL language. However, OWL has some defects in the description of the rough ontology and cannot describe the nondiscrimination relation and the concept of the upper and lower approximation set. OWL cannot establish the rules in general field by itself, so it cannot excavate implied relationship between the social relations of ontology.

SWRL [10] is based on OWL DL and OWL Lite, and it introduces the regular descriptive method of RuleML (Rule Markup Language) in order to enhance the ability of rule description and inference in the case of the strong expressive ability of knowledge by using OWL. At the same time, SWRL is based on the logic of first-order predicate logic, and it can infer implied knowledge from the knowledge that has already existed. Although SWRL has the stronger ability for semantic expression, the knowledge which is expressed by SWRL is precise. The uncertainty of information is prevalent 
in real life; SWRL cannot satisfy the need to express uncertain knowledge.

There are not many studies on the language of SWRL at present. Yiduo [11] researches the formal representation of fuzzy ontology and enhances the expressive ability of fuzzy rules in the semantic Web. Wang et al. [12] introduce the extensional form of vague SWRL based on fuzzy SWRL of vague set, and study on the syntax of vague SWRL, and give the rule examples of vague SWRL. Pan et al. [10] put forward the extensional form of f-SWRL which is based on fuzzy set of SWRL for the first time. However, it is difficult to express the uncertain or incomplete knowledge which is universal by the method based on the fuzzy value of membership.

The theory of rough set was given by Professor Pawlak in 1982; it is a mathematical tool that can analyze and deal with the uncertain or incomplete information quantitatively, and it is a discovery which achieved the approximate knowledge through the classification of equivalent relations [13]. In order to enhance the expressive ability of uncertain knowledge, this paper uses the extension for SWRL based on the theory of rough set, builds RSWRL (Rough Semantic Web Rule Language), and extends reasoning rules.

\section{Rough Set and Rough Ontology}

\subsection{Rough Set}

Definition 1. Approximate space can be expressed as $K=(U$, $R)$. Among them, $U \neq \Phi$ is a nonempty finite set, and it is called domain; $R$ is an equivalent relation on $U$.

Definition 2. $U \neq \Phi$ is a nonempty finite set; $R$ which is one of the relations of $U$ is called the equivalent relation on $U$, only when it is a partition $\left\{X_{1}, X_{2}, \ldots, X_{n}\right\}$ of $U$ based on $R$, $X_{i} \subseteq U, X_{i} \neq \Phi$, and $X_{i} \cap X_{j}=\Phi, i \neq j, i, j=1,2, \ldots, n$; $i=1 n X_{i}=U$. Different partition corresponds to different subset (class) of $U$; a family which is divided on $U$ is called a knowledge base on $U ;[x]_{R}$ expresses the equivalent class $R$ that contains the element $x \in U$.

Definition 3. Set $x \subseteq U, R$ is an equivalent relation of $U$. When $X$ can be expressed to the union of some basic sets $R$, it is said that $X$ can be defined on $U$; otherwise it is called that $X$ cannot be defined on $U . R$ can be defined as a subset of domain $U$, and it can be precisely defined in the knowledge base $K$; it is called accurate set $R$ (an empty set is also called definable set). But definition set $R$ cannot be precisely defined in the knowledge base $K$; it is called inexact set $R$ or rough set $R$ (rough set). Rough set can be defined approximately; that is to say, it can be described by two precise sets (upper approximation and lower approximation of rough set) "similarly."

Definition 4. Set knowledge base $K=(U, R)$, and $X$ is any subset of $X, R$ is equivalent relation, and then the upper and lower approximations of $X$ can be represented as follows:

$\operatorname{apr} X=\underline{R} X=\left\{x \mid x \in U,[x]_{R} \subseteq X\right\}$; it is called that $\overline{\text { set }} X$ is related to the lower approximation of $R$. $\overline{\operatorname{apr}} X=\bar{R} X=\left\{x \mid x \in U,[x]_{R} \cap X=\varnothing\right\} ;$ it is called that set $X$ is related to the upper approximation of $R$.

2.2. Rough Ontology. Perez and Benjamins give a five-tuple [14], which consists of concept, relationship, function, axiom, and example. Because the function of psychological counseling case can be described by relation, the ontology of psychological counseling case can be described formally as $O:=\langle C, R, A, I\rangle$; among them, $C$ is a concept or class, and it is a set of objects with common properties; $R$ is the order relationship between the concepts; $A$ is the axiom of ontology; $I$ is a set of ontology examples.

In the practical substance rough set of ontology uses the established concepts that have already existed to express the unknown and imprecise concepts. That is to say, the concept consists of extension and connotation, the extension of concept represents the objects that are covered by concepts, including the objects that may be covered or must be covered, and they are corresponded with upper and lower approximations of rough set; the connotation represents the common properties of objects that are covered by concepts. The theory of rough set is introduced on the basis of ontology concept, and it forms rough ontology. It can not only retain the extensible advantage of ontology structure, but also enhance the expressive ability of ontology technology for uncertain knowledge. Therefore, the rough ontology is defined as follows.

Definition 5. Rough ontology can be expressed by a tetrad: $O:=\left\langle C_{R}, R_{R}, A_{R}, I_{R}\right\rangle$, where $C_{R}$ represents the set of rough concepts, $R_{R}$ represents the rough relationship between rough concepts, $A_{R}$ represents the ontology axiom based on the theory of rough set, and $I_{R}$ represents the rough example.

Rough ontology is the extension of ontology theory for rough set, the concept is rough concept, the relationship is rough relationship between the rough concepts, and the example is rough example.

\section{The Semantics of RSWRL}

SWRL FOL is based on predicate logic, the concept $C$ of SWRL is no longer a precise definition, and it is explained as a rough subset. RSWRL just makes the extensions of the concepts; the axiom of RSWRL has the same grammar with SWRL. That is to say, the axiom does not need the extension of ontology axiom to be obtained. So this paper just gives the extensions for classes, properties, and examples of RSWRL.

Definition 6. The semantic explanation of RSWRL is a binary form: $I=\left(\Delta^{I}, I\right) ; \Delta^{I}$ is a nonempty set and $\cdot I$ is a function of the rough explanation.

The rough explanation of function is defined as follows:

(i) The concept of top: $T$ represents everything; it is the same with the explanation of SWRL.

(ii) The concept of bottom: $\perp$ represents empty; it is the same with the explanation of SWRL. 
(iii) The explanation for individual constants: individual constants are mapped to the elements of $\Delta^{I}$; it is the same with the explanation of SWRL.

(iv) The explanation for concept: the concept $C$ is mapped to the difference between the upper and lower approximation of $\Delta^{I}$; that is to say, $C^{I}: \Delta^{I} \rightarrow \bar{C}-\underline{C}$.

(v) The explanation for relationship: relation $R$ is mapped to the relation between approximate sets of $\Delta^{I}$; that is to say, $R^{I}: \Delta^{I} \times \Delta^{I}$, except the set is rough set; the others are the same with the explanation of SWRL.

(vi) The explanation for modified operator: the imprecise concept is described by accurate concept; it can extend SWRL by adding “"” and ".” two operators. They correspond to upper and lower approximations of rough set to express the sets which may belong to or must belong to some concept.

(vii) The explanation for assertion: if $C$ and $D$ are the concept terms of rough description logic, then there are main forms for the collection of assertions:

(1) $C \subseteq D$; it is called that rough description contains assertion.

(2) $C \equiv D$; it is called that rough description is equivalent with assertion.

\section{The Definition of the Language RSWRL}

4.1. The Extension for Classes of RSWRL. The classes of SWRL are divided into simple classes (or basic classes) and complex classes. Simple classes are described by using name and limiting for basic concepts. Complex classes are the classes that are constructed by using some constructors (such as acquaintances, unions, and complements) of some simple classes, and the purpose is to extend the expressive ability of SWRL, so this paper only defines rough simple classes (or rough classes), and rough complex classes can be obtained by the structure of simple rough classes.

\subsubsection{The Definition of Rough Class}

Definition 7. The rough concept is a triple [15]: $C_{R}:=\langle\{U$, $L\}, I\rangle .\{U, L\}$ is the extension of the rough concept $C_{R}$, and $I$ is the connotation of the rough concept $C_{R}$, and it is generally represented by rough membership. $U$ and $L$ correspond to the upper approximation set and lower approximation set of the rough concept $C_{R}$. The former represents all the sets of objects that may be covered; the latter represents all the sets of objects that must be covered.

The expressive method of rough ontology in RSWRL is to add "up-class" and "low-class" in the label of ObjectProperty, and they represent, respectively, the classes that they "may" and "must" belong to, to add "up-property" and "low-property," and they represent the properties that they "may" and "must" have, to add "precise" which represents approximation in the ObjectProperty at the same time. The format of RSWRL also uses the same way with the format of OWL as the rules appeared to be designed, the biggest benefit is that the internal variables are represented by the ways of $\mathrm{RDF} / \mathrm{XML}$, and it makes the variables that are corresponding to the ontology more relatively simple. The work of this paper will be based on the syntax format of RDF.

Example 8. The description of symptoms about panic disorder in psychological counseling field may have two situations: \{hot and dry, chest pain, flustered, hands and feet numbness, tremble, upset, nervous and afraid\} and \{flustered, nervous and afraid\}, and it may be caused by different understandings for the symptoms of panic disorder. But the symptoms of "panic disorder" must include being "flustered, nervous, afraid," and it may have the symptoms like "hot and dry, chest pain, hands and feet numbness, tremble, upset, etc." at the same time; then it represents the language of RSWRL on the basis of RDF/XML format by the above method as follows:

$$
\begin{aligned}
& \text { <rswrl:Class rdf:ID="panic disorder"> } \\
& \text { <rswrl:ObjectProperty rdf:ID="up-property"> } \\
& \text { <rswrl:hasValue rdf:resource="hot and dry, } \\
& \text { pain, flustered, hands and feet numbness, trem } \\
& \text { upset, nervous and afraid"/> } \\
& \text { </rswrl: ObjectProperty }> \\
& \text { <rswrl:ObjectProperty rdf:ID="low-property"> } \\
& \text { <rswrl:hasValue rdf:resource="flustered, nervou } \\
& \text { afraid"/> } \\
& \text { </rswrl: ObjectProperty > } \\
& \text { <rswrl:ObjectProperty rdf:ID="precise"> } \\
& \text { <rswrl:hasValue rdf:resource="3/8"/> } \\
& \text { </rswrl: ObjectProperty }> \\
& \text { </rswrl:Class }>
\end{aligned}
$$$$
<\text { rswrl:hasValue rdf:resource="hot and dry, chest }
$$$$
\text { pain, flustered, hands and feet numbness, tremble, }
$$$$
<\text { rswrl:hasValue rdf:resource="flustered, nervous and }
$$

4.1.2. The Definition of Subclass and the Inclusion of Class. The definition of class of SWRL contains class, subclass, the intersection of classes, the equivalence of classes, and so on. It is the same with the class of SWRL, and all the classes of RSWRL are the subclasses of Thing.

Definition 9. Class identifier rswrl: subClassOf states the rough subclass of RSWRL; its logical grammar is $B \leftarrow A$; it represents the fact that $A$ is a subclass of $B$.

Example 10. "Depression" is a kind of "mental illnesses," and there is an inheritable relationship between "depression" and "mental illnesses." It represents the language of RSWRL on the basis of RDF/XML format as follows:

$$
\begin{aligned}
& <\text { rswrl:Class rdf:ID="mental illnesses" }> \\
& </ \text { rswrl:Class }> \\
& <\text { rswrl:Class rdf:ID="depression" }> \\
& \quad<\text { rswrl:subClassOf } \\
& \text { rdf:resource="depression" } /> \\
& </ \text { rswrl:Class }>
\end{aligned}
$$




\subsubsection{The Definition of the Equivalent Classes}

Definition 11. Class identifier rswrl: equivalentClass states the equivalent classes of RSWRL; its logical grammar is $A \equiv B$; it represents the fact that $A$ is equivalent to $B$.

Example 12. The class of "mental illness" and the class of "psychological disorder" are equivalent. It represents the language of RSWRL on the basis of RDF/XML format as follows:

$$
\begin{aligned}
& <\text { rswrl:Class rdf:ID="mental illness" }> \\
& <\text { rswrl:equivalentClass } \\
& \text { rdf:resource="\#psychological disorder"/> } \\
& <\text { /rswrl:Class }>
\end{aligned}
$$

4.1.4. The Definition of Rough Complex Class. The basic class of RSWRL can generate complex class through the structures like union, intersection, complement, enumeration, and so on between classes.

Example 13. In psychological consulting field, it is generally believed that the patient who has the following symptoms can be considered as a patient who has "panic disorder": "flustered, nervous and afraid; hot and dry, chest pain, upset, hands and feet numbness, tremble." It represents the language of RSWRL on the basis of RDF/XML format as follows:

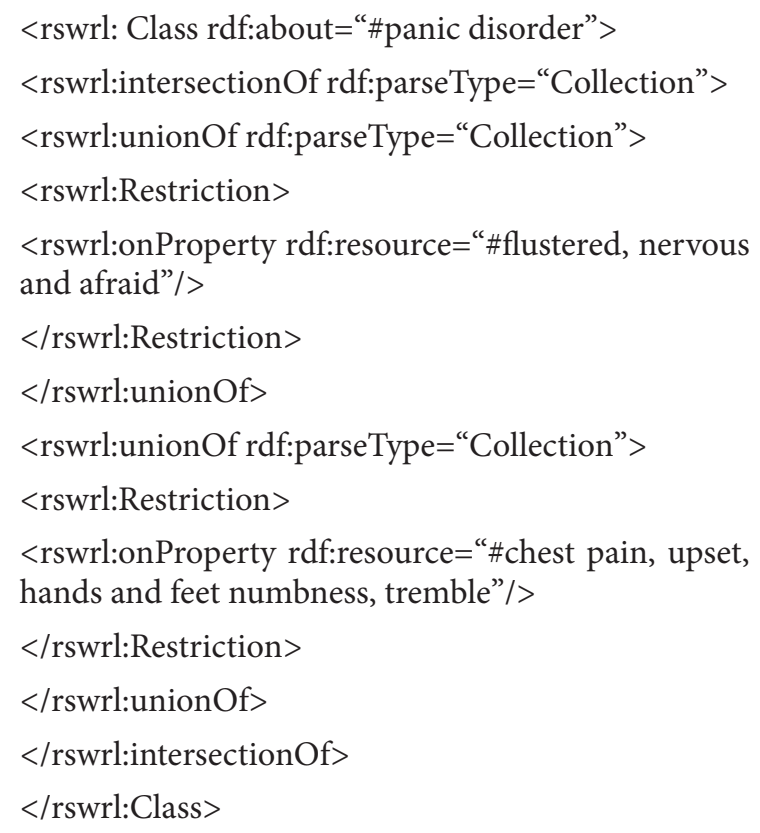

4.2. The Extension of the Properties of RSWRL. In addition to the transitivity, symmetry, and the properties of function, the declaration for the properties in SWRL also includes the constraint of the full name and the constraint of the existence.

Properties are divided into two kinds: one is "data type properties." They are used to define the inherent properties of a class. For example, when we define the property of "patient" in the field of psychological counseling, the name, age, gender, and other data type properties of the patient should be defined. Another kind is "object properties." They are the associated properties between the two classes; for example, the object properties of rough ontology in the field of psychological counseling can fully express the relationships between the instances of concepts, and they are the foundations of knowledge reasoning. For example, the domain of property "Has_Symptom" is Disorder_Type, the range is "Symptom_Criteria," and they explain the relationship between the types and symptoms of the disorders.

RSWRL inherits the basic expressing ability of the properties of SWRL and expands the similar expression of relationship.

Definition 14. Identifier rswrl: similarPropertyTo states the similar relationship between the properties of RSWRL, its logic grammar is: similarProperty $(A, B)$, and it represents the fact that $A$ is similar to $B$.

Example 15. The relationship between psychological counselors and patients in the process of psychological counseling can not only say that psychological counselors "cure" the patients, but also say that psychological counselors "treat" the patients; that is to say, "cure" and "treat" are similar binary relation in the psychological counseling field. The above content is expressed by using RSWRL based on RDF/XML format as follows:

$$
\begin{aligned}
& \text { <rswrl: ObjectProperty rdf:ID="cure" > } \\
& \text { <rswrl: similarPropertyTo rdf:resource="\#treat"/> } \\
& \text { <rswrl:contextOf rdf:ID="S"> } \\
& \text { <rswrl:basicClass rdf:about="\#psychological coun- } \\
& \text { seling"/> } \\
& \text { </rswrl:contextOf }> \\
& \text { </rswrl: ObjectProperty }>
\end{aligned}
$$

4.3. The Extension of RSWRL Truth. The main forms of the description for truth in SWRL are the examples and axioms of classes, and the examples mainly refer to the instantiations of classes and relations. This paper mainly describes the truth of ontology by the extension of classes and relations of RSWRL.

Definition 16. Identifier rswrl: Individual states the individuals of RSWRL.

Example 17. There are two psychological counselors in a psychological counseling clinic, they are Zhang San and Li Si, and their roles are the same. In other words, they are similar in the process of psychological counseling, and this relation can be expressed by RSWRL based on RDF/XML format as follows:

$$
\begin{aligned}
& \text { <rswrl: Thing rdf:ID=“Zhang San"> } \\
& \text { <rswrl: type rdf:resource="\#physical therapist"/> } \\
& \text { </rswrl: Thing }> \\
& \text { <rswrl: Thing rdf:ID="Li Si”> } \\
& \text { <rswrl: SimilarIndividual > } \\
& \text { <rswrl: Thing rdf:ID="Zhang San"> }
\end{aligned}
$$


$</$ rswrl: SimilarIndividual $>$

$<$ rswrl:contextOf rdf:ID="S">

$<$ rswrl: basicClass rdf:about="\#psychological counseling"/>

$</$ rswrl:contextOf $>$

$</$ rswrl: Thing $>$

4.4. The Extension of RSWRL Rules. Although the semantic expressive ability of OWL is better than RDF, it is mainly based on concepts, classifications, and axioms, and it has certain limitations, such as it cannot define the concept "Uncle: Parent(?x, ?y) $\wedge$ Brother(?y, ?z) $\rightarrow$ Uncle(?x, ?z)" by using first-order logic. In order to make up for the defects of the rules in OWL, the rules of SWRL [16] expand the logical expressive ability of OWL. SWRL is built on the basis of OWL, which can be combined with the classes and the relationships of OWL ontology. It can not only express the certain information, but also express the rules of imprecise information. According to the reasoning rules, the information of implicit semantics is realized, which can improve the efficiency of reasoning.

SWRL is mainly composed of 4 parts, and they are, respectively, Imp, Atom, Variable, and Built-in [17]. Imp is composed of SWRL rule, "head" shows the result of reasoning in Imp, and "body" shows the basic form of reasoning precondition. The basic component of head is "Atom" in Imp; "Variable" is used to record the variables used in Atom; "Builtin" is a modularized component of SWRL, and it records the relationship of logical comparison which can be referred to in SWRL. The general form is Antecedent-Consequent [18].

According to the rules of SWRL, the RSWRL rules based on rough concept can be constructed. The simple classes, complex classes (intersection, union, complement, and equivalent set) can directly correspond to the rough classes of RSWRL. At the same time, RSWRL also inherits the basic expressive ability and reasoning ability of SWRL, and it extends the expression of similar relationship. Accordingly it basically covers the main forms of grammar that have already existed in SWRL, and it can define and express the uncertain semantic knowledge based on SWRL to carry out the knowledge inference of rough ontology.

\section{The Examples of Application}

5.1. The Ontology Model of Psychological Counseling Case. Case-Based Reasoning (CBR) is a set of methods that has a long tradition in the field of artificial intelligence. With the help of past experiences, old cases, ratiocination, and the mechanism learning to solve problems, Case-Based Reasoning involves the use of certain methods in memory or past case base to find the issue of the same or most similar source cases and reuses the solution to the original problem in the new cases.

Figure 1 is an ontology model of panic disorder case; it describes some fragments of knowledge that are caused by the event of panic disorder.
With the consolidation of knowledge for the event of panic disorder, it is classified according to the structure of knowledge; some results are shown in Table 1.

5.2. The Representation of Psychological Counseling Cases Based on RSWRL. Panic disorder (PD) is an acute anxiety disorder that appears as repeatedly obvious plant nerve symptoms, including palpitation, sweating, and tremor, and it is distinguished by the panic seizure of unfortunate consequences. The knowledge in Table 1 can be represented by RSWRL based on RDF/XML format; for space reasons, only the fragments of knowledge in RSWRL for the event of panic disorder are given as follows:

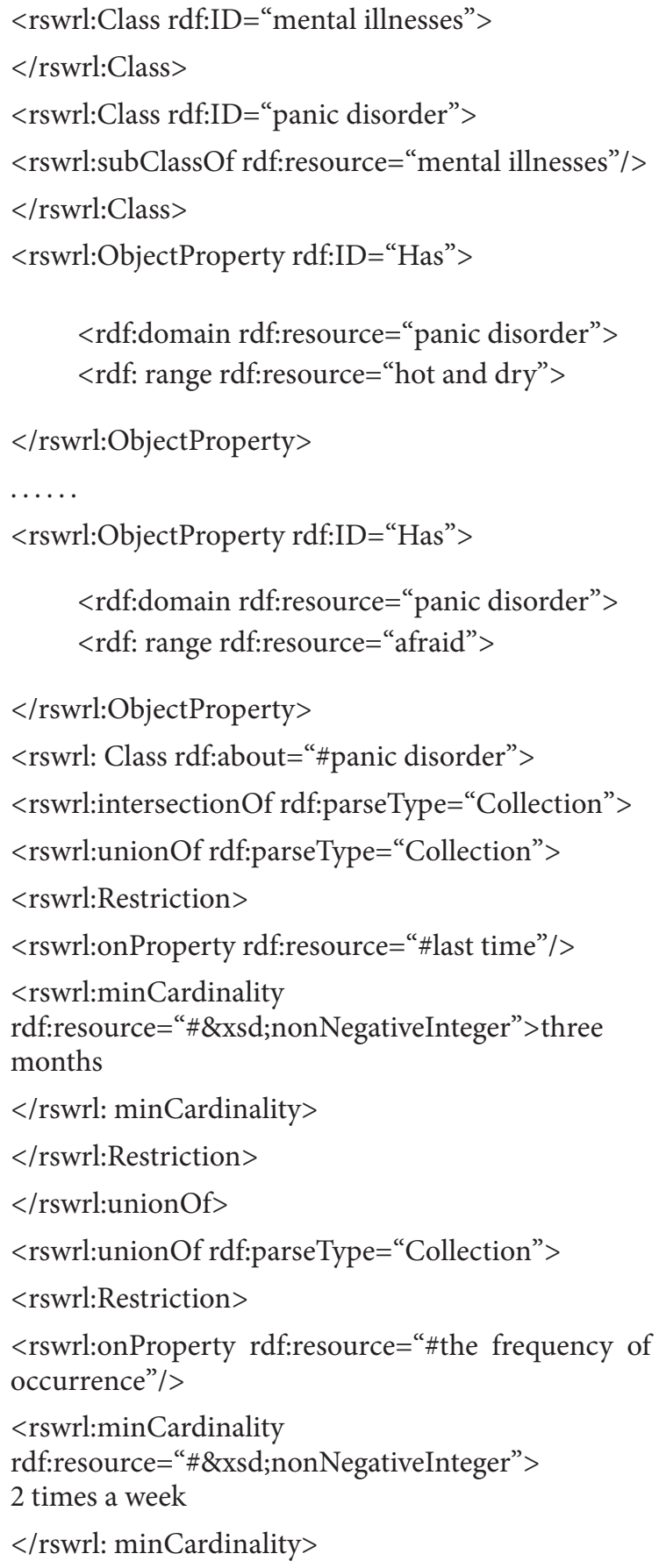




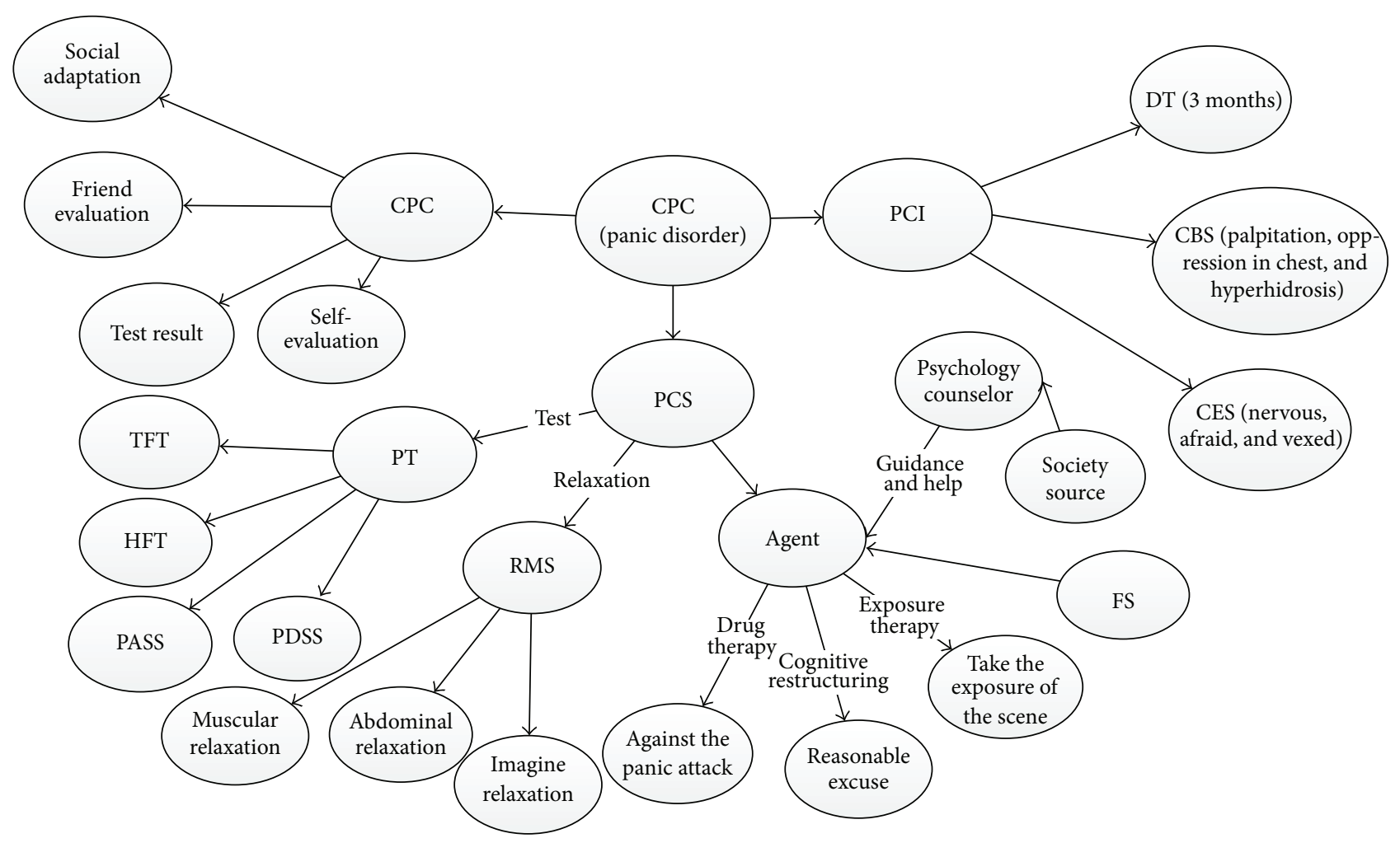

Explanation:

CPC: CBR-based psychology case

PCI: the information of psychology case

DT: the time of duration

CBS: behavioral symptoms of case

CES: emotional symptoms of case
PCS: the solution of psychology case
PT: psychology test
TFT: thyroid function test
HFT: heart function test
PASS: panic-associated symptom scale PDSS: panic disorder severity scale RMS: the study of relaxation method FS: the support of friends

FIGURE 1: The fragments of knowledge that are caused by the event of panic disorder.

TABLE 1: The knowledge classification of psychological counseling cases.

\begin{tabular}{|c|c|c|}
\hline \multicolumn{2}{|c|}{ Key elements } & Keywords \\
\hline \multirow{3}{*}{ Example concept } & Psychological events & Panic disorder \\
\hline & Event state & Last for 3 months, palpitation, oppression in chest, nervous, afraid \\
\hline & Social resources & Relatives, friends, social support system \\
\hline \multirow{3}{*}{ Relations concept } & Compose relationship & Compose \\
\hline & Have relationship & Target \\
\hline & Action relationship & Treatment, cognize, relax, test \\
\hline
\end{tabular}

\section{$</$ rswrl:Restriction $>$}

$</$ rswrl:unionOf $>$

$<$ rswrl:unionOf rdf:parseType="Collection" $>$

$<$ rswrl:Restriction $>$

$<$ rswrl:onProperty rdf:resource="\#upset, nervous, afraid"/>

$</$ rswrl:Restriction $>$

$</$ rswrl:unionOf $>$

$<$ rswrl:unionOf rdf:parseType $=$ "Collection" $>$

$<$ rswrl:Restriction $>$
$<$ rswrl:onProperty rdf:resource="\#chest pain, upset, hands and feet numbness, tremble"/>

$</$ rswrl:Restriction $>$

$</$ rswrl:unionOf $>$

$</$ rswrl:intersectionOf $>$

$</$ rswrl:Class $>$

5.3. The Reasoning Based on RSWRL. At present, the recognition rate is relatively low for panic disorder, and it often has not enough consideration with improper treatment. The patients and doctors often misdiagnosed it as angina pectoris, 
and it increases the patients' mental burden, and they do not get the effective treatment. In the clinical work, many unexplained symptoms in medical science may be a sign of panic attacks. When there is a patient in a panic attack, but there is not the feeling of fear, it can just be described as an "extreme discomfort" [19]. So there are no specific diagnostic criteria for panic disorder, and it is even more difficult to make the correct diagnosis.

In order to avoid misdiagnosis and missed diagnosis, when the patients describe inaccurate or incomplete symptoms by themselves, we can have a diagnosis scheme for panic disorder through the inference rules as follows.

Rule 1. Patient $(? \mathrm{x}) \wedge$ Panic_Disorder(?y) $\wedge$ Has_symptom $(? \mathrm{x}$, upset $) \wedge$ Has_symptom(?x, nervous $) \wedge$ Has_symptom(?x, elevation of blood pressure) $\rightarrow$ Has_Panic(?x, ?y).

The semantic content expressed by Rule 1 is as follows: Patients X: if there are some symptoms such as being upset and nervous and the elevation of blood pressure, the patient may be suffering from panic disorder.

Rule 2. Patient $(? \mathrm{x}) \wedge$ Panic_Disorder $(? \mathrm{y}) \wedge$ Has_symptom $(? \mathrm{x}$, dizziness $) \wedge$ Has_symptom(?x, fever pitch $) \wedge$ Has_symptom(?x, dyspnea) $\rightarrow$ Has_Panic(?x, ?y).

The semantic content expressed by Rule 2 is as follows: Patients X: if there are some symptoms such as dizziness, fever pitch, and dyspnea, the patient may be suffering from panic disorder.

Rule 3. Patient $(? \mathrm{x}) \wedge$ Panic_Disorder(?y) $\wedge$ Has_symptom(?x, a sense of fear $) \wedge$ Has_symptom $(? x$, sense incontrle $) \wedge$ Has symptom(?x, depersonalization) $\rightarrow$ Has_Panic(?x, ?y).

The semantic content expressed by Rule 3 is as follows: Patients X: if there are some symptoms such as a sense of fear, sense in control, and depersonalization, the patient may be suffering from panic disorder.

Through the extension of SWRL, this paper defines the rough ontology description language RSWRL, and it can better express the uncertainty of knowledge in psychological consultation. It extends the rules by combining with the knowledge of psychological counseling to realize the reasoning of knowledge, and it perfects the content of the knowledge base of ontology.

\section{Conclusion}

This paper introduces the theory of rough set and gives the semantic interpretation of RSWRL on the basis of rough ontology. Then it extends and defines RSWRL; finally it describes a psychological counseling case by using RSWRL. Because OWL DL is not able to fully express some complex rules, through the extension of SWRL, the rules of reasoning that are used in the psychological counseling cases are put forward. The result shows that RSWRL can well express the imprecise knowledge, and the new ontology has more abundant semantic relation between the rough concepts for the inference of knowledge, and the content of ontology knowledge base is improved. In the following research, we will focus on the study of retrieval method based on rough ontology and establish the corresponding inference rules according to the demand. It can not only improve the rate of utilization of ontology knowledge, but also enhance the recall and precision when the research results will be applied for the retrieval of information.

\section{Conflict of Interests}

The authors declare that there is no conflict of interests regarding the publication of this paper.

\section{References}

[1] Y. Lv, Z. Ma, and F. Zhang, "The construction method of fuzzy ontology based on fuzzy conceptual model," Journal of Northeastern University (Natural Science Edition), vol. 30, no. 9, pp. 1262-1265, 2009.

[2] K. Dazhou, X. Baowen, and L. Jianjiang, "Description logic for fuzzy ontology on semantic web," Journal of Southeast University, vol. 22, no. 3, pp. 343-347, 2006.

[3] L. Haiyan, Research on knowledge reasoning supported by rough ontology of semantic web [M.S. thesis], Dalian Maritime University, Dalian, China, 2012.

[4] H. Xiaomin, The Research on Fuzzy Rough Ontology Reasoning of Semantic Web, Dalian Maritime University, 2013.

[5] L. Xiaoyin, The ImPrecise ontology modeling and its construction [Ph.D. thesis], Dalian Maritime University, Dalian, China, 2011.

[6] H. Xiaomin and L. Guanyu, "Fuzzy rough ontology driven reasoning machine research," Computer Engineering and Design, vol. 34, no. 10, pp. 3619-3624, 2013.

[7] W. Hailong, M. Zongmin, Y. Li, and Y. Dongliang, "A fuzzy extension of OWL based on F-ALC (G)," Journal of Northeastern University (Natural Science Edition), vol. 30, no. 11, pp. 1562$1565,2009$.

[8] W. Hongyun, Research on Knowledge Acquisition Method Supported by Semantic Web Rough Ontology, Dalian Maritime University, Dalian, China, 2012.

[9] S. Xijin, Research on semantic web rough fuzzy ontology and its application [Ph.D. thesis], Dalian Maritime University, Dalian, China, 2012.

[10] J. Z. Pan, G. Stoilos, G. Stamou et al., "f-SWRL: a fuzzy extension of SWRL," in Journal on Data Semantics VI, vol. 4090 of Lecture Notes in Computer Science, pp. 28-46, Springer, Berlin, Germany, 2006.

[11] L. Yiduo, Knowledge representation for fuzzy system on the semantic web [Ph.D. thesis], Dalian Maritime University, Dalian, China, 2013.

[12] X. Wang, Z.-M. Ma, and X.-F. Meng, "Fuzzy extension of semantic web rule language," Journal of Northeastern University (Natural Science Edition), vol. 30, no. 5, pp. 632-635, 2009.

[13] Z. Pawlak, "Rough sets," International Journal of Computer \& Information Sciences, vol. 11, no. 5, pp. 341-356, 1982.

[14] A. G. Perez and V. R. Benjamins, "Overview of knowledge sharing and reuse components: ontologies and problem-solving methods," in Proceedings of the 16th International Joint Conference on Artificial Intelligence (IJCAI '99) Workshop KRR5: Ontologies and Problem-Solving Methods: Lesson Learned and Future Trends, pp. 1-15, Stockholm, Sweden, August 1999. 
[15] W. Dongyan, A method for building semantic web rough ontology [M.S. thesis], Dalian Maritime University, Dalian, China, 2011.

[16] L. Bellatreche, N. X. Dung, G. Pierra, and D. Hondjack, "Contribution of ontology-based data modeling to automatic integration of electronic catalogues within engineering databases," Computers in Industry, vol. 57, no. 8-9, pp. 711-724, 2006.

[17] D. Shengchun and J. Chaonan, "The mining of the implicit relations based on SWRL reasoning," Modern Technology of Library and Information, no. 3, pp. 68-72, 2011.

[18] J. Zhaohui and L. Cunhua, "The web rules based on SWRL andjess and the analysis of countermeasure," Journal of Huaihai Institute of Techology: Natural Science Edition, vol. 12, 2009.

[19] T. Y. Qiong, "The 26 cases of misdiagnosis analysis on panic disorder," Chinese Journal of Misdiagnosis in Learning, no. 27, article 6661, 2010. 


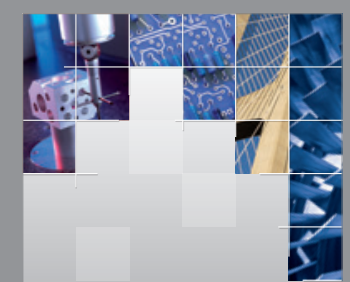

\section{Enfincering}
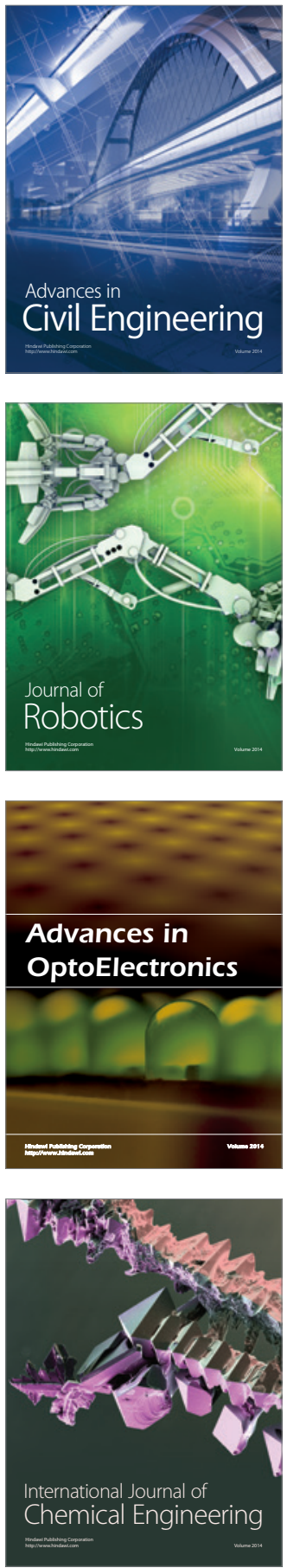

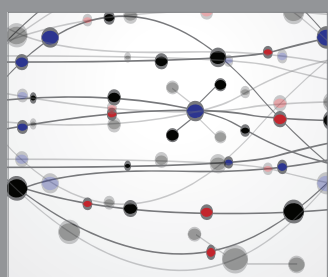

The Scientific World Journal

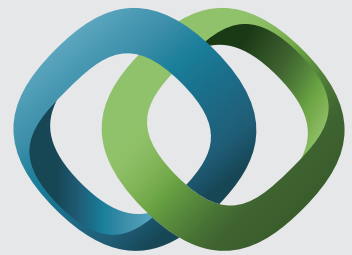

\section{Hindawi}

Submit your manuscripts at

http://www.hindawi.com
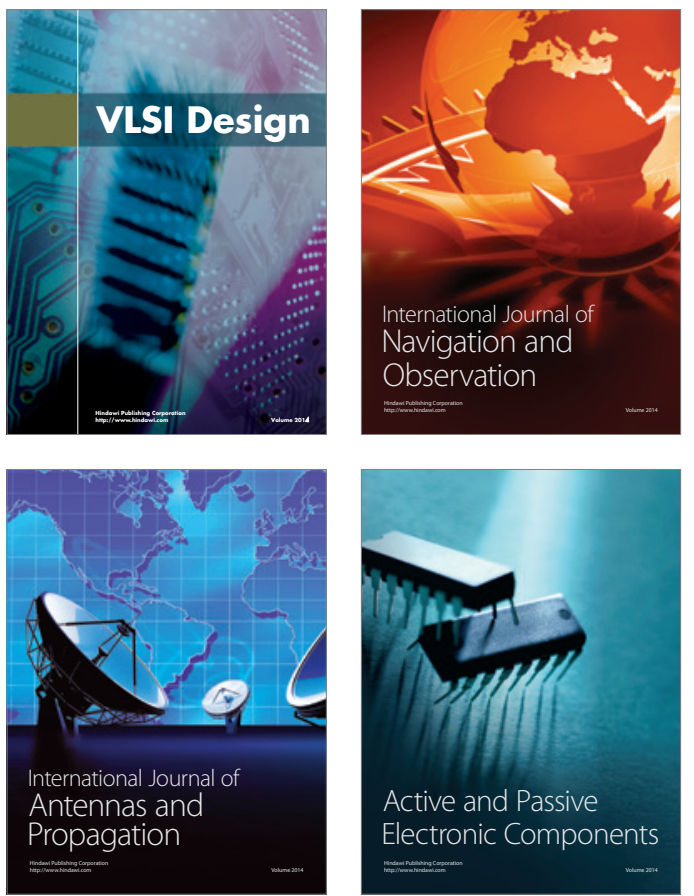
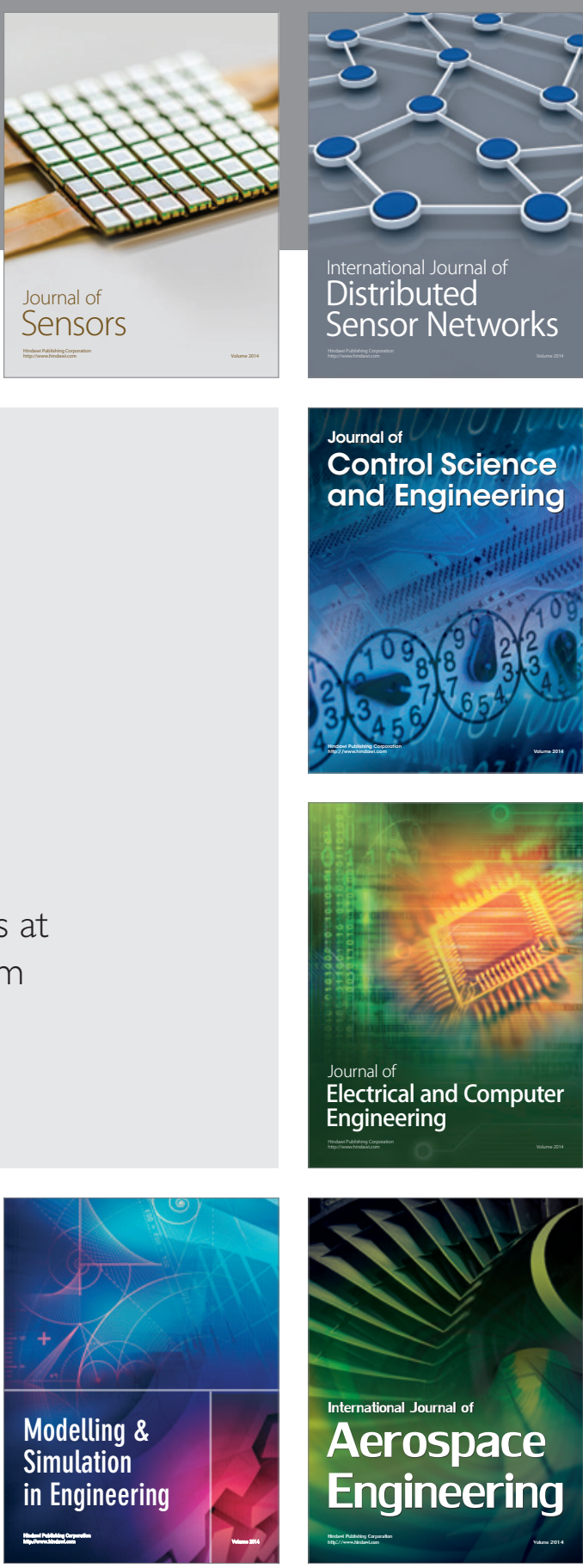

International Journal of

Distributed

Sensor Networks

Journal of

Control Science

and Engineering
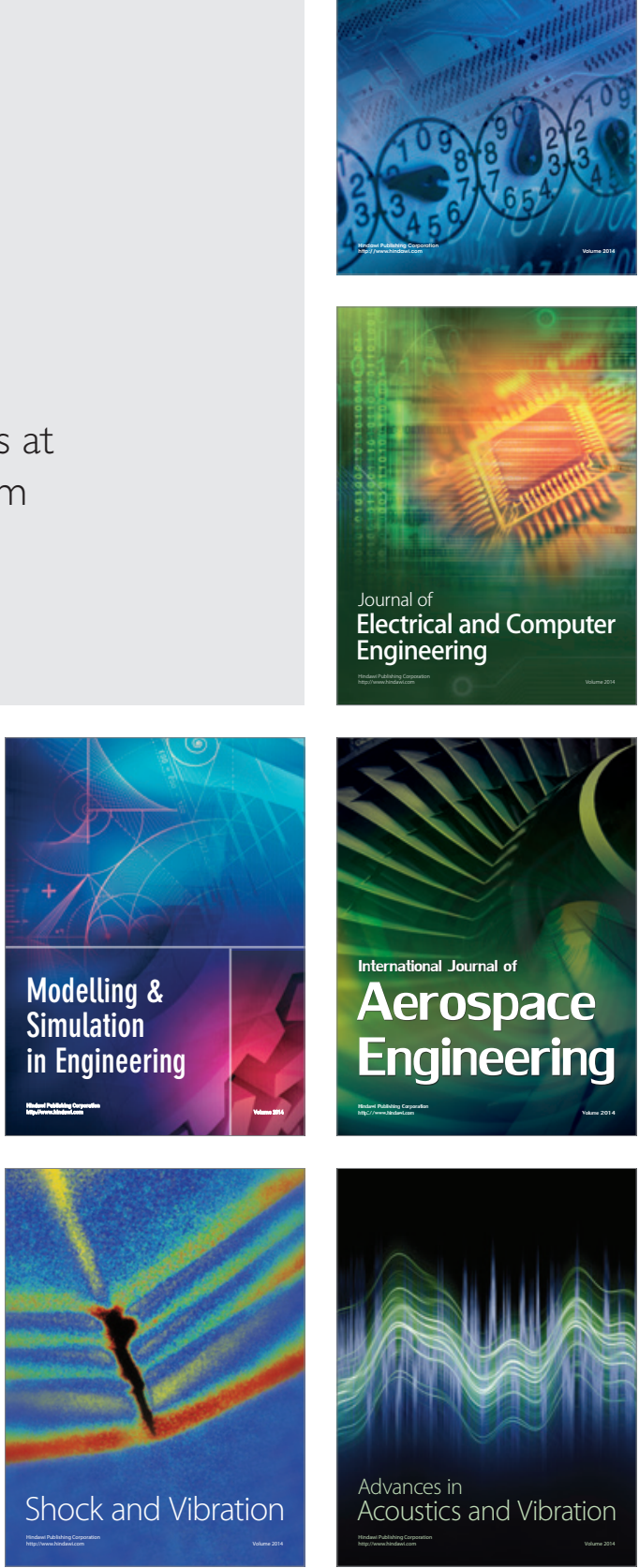